\section{The microbial carbon pump and the oceanic recalcitrant dissolved organic matter pool}

\author{
Nianzhi Jiao, Gerhard J. Herndl, Dennis A. Hansell, Ronald Benner, \\ Gerhard Kattner, Steven W. Wilhelm, David L. Kirchman, Markus G. Weinbauer, \\ Tingwei Luo, Feng Chen and Farooq Azam
}

We discuss below the correspondence relating to our Opinion article about the 'microbial carbon pump' (MCP) (Microbial production of recalcitrant dissolved organic matter: long-term carbon storage in the global ocean. Nature Rev. Microbiol. 8, 593-599 (2010)) ${ }^{1}$, by Chen (Microbial carbon pump: additional considerations. Nature Rev. Microbiol. 31 May 2011 (doi:10.1038. nrmicro/2386-c4) $)^{2}$. The MCP refers to the mechanisms of microbial transformation of labile dissolved organic matter (LDOM) to recalcitrant dissolved organic matter (RDOM). RDOM is resistant to biological degradation, can remain in the water column for long periods of time and can thereby sequester carbon in the ocean. The concept of the MCP is also applicable in soils and other environments ${ }^{3,4}$. However, the MCP mechanism (carbon transformation) and its effect (carbon sequestration in the dissolved organic phase) are not interpreted by Chen as we had intended. According to his interpretation of the MCP concept, "DOM in the surface layer of the oceans comprises only LDOM".

This suggestion stems from an interpretation of figure 2 in our article ${ }^{1}$ to mean that there is an absence of RDOM in the surface ocean. Such an absence was not intended to be implied by this or any other figure in our article, as RDOM is in fact present throughout the ocean water column. Indeed, our text explicitly defines RDOM as "the major fraction of DOM found throughout the entire water column". Figures 2 and 4 do not repeat this information, but instead depict the microbial transformation of carbon from LDOM to RDOM; these figures depict 'effects' and 'mechanisms', respectively, and not quantities or distributions (note the absence of scale on the $x$-axes in the figures).

The second point raised by Chen regarding fossil DOM is not relevant to the MCP. The goal of our article was to consider how microbial processes transform the photosynthetically fixed carbon from the surface ocean through the water column. We did

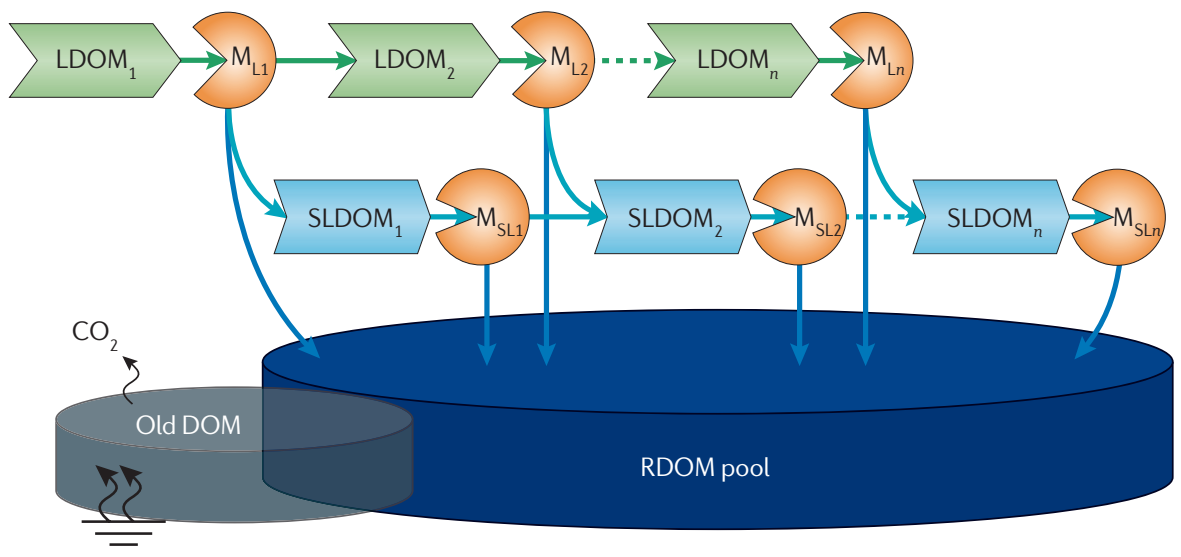

Figure 1 | The successive microbial carbon pump processes for recalcitrant dissolved organic matter (RDOM) formation and subsequent contribution to the oceanic RDOM pool. $M_{L}$ and $M_{S L}$ represent those microorganisms that can use labile dissolved organic matter (LDOM) or semi-labile DOM (SLDOM), respectively. The subscript numbers indicate the numerous compounds or microbes. Extents of recalcitrance are indicated by the colour sequence dark blue > light blue > green; grey indicates old DOM from seabed seeps or hydrothermal vents, which is not entirely recalcitrant, as some of these old compounds are readily available to microorganisms for respiration. Note that multiple processes are simplified here (for example, the back flows from SLDOM to LDOM). not seek to quantify all carbon fluxes or to address the age of the RDOM. However, we did recognize the DOM that is introduced to the water column from hydrothermal vents or seabed seeps (see the lower right-hand corner of figure 4 in our article). Further consideration of the contribution of these fluxes was, unfortunately, beyond the scope and intent of our article.

In our article, we emphasize the linkage between the products of the MCP and the oceanic RDOM pool. Defined from a biological point of view, RDOM should have no time dimension. If a bacterium does not have a gene encoding an enzyme for a corresponding substrate, it cannot use the substrate regardless of timescale; the molecule is forever recalcitrant to that bacterium. This reasoning is true even at functional group or taxon levels. However, material that is recalcitrant to one microbial group can be available to another. Hence, we emphasized "the need to understand the ability of microorganisms to process DOM at various taxonomic and functional-group levels". In practice, diverse microbial processes could be involved in the formation of RDOM, and environmental conditions could also influence the recalcitrance of DOM molecules ${ }^{5,6}$. It is the successive microbial processing of LDOM and semi-labile DOM that finally forms the RDOM compounds which contribute to the oceanic RDOM pool characterized by great radiocarbon age (FIG. 1). This carbon pool is also introduced by other old carbon sources such as hydrothermal vents and seabed seeps ${ }^{7}$. However, these other forms of old DOM are not entirely recalcitrant; some of this DOM is readily available for biological utilization and degradation ${ }^{8}$. Therefore, the recalcitrance of DOM cannot simply be defined by age; it can be defined structurally (using molecular characteristics, for example) or experimentally (using bioassays) ${ }^{9}$.

We proposed the MCP as a "conceptual framework to address the role of microbial generation of RDOM", but we did not intend to quantify fluxes. However, in view of the multiple sources and mechanisms of microbial processing for a large proportion of fixed carbon, we predicted that the MCP is probably a quantitatively important source of RDOM. It was recently estimated that RDOM of bacterial origin (one of the MCP pathways) constitutes 155 Gigatonnes of carbon from the total oceanic RDOM pool ${ }^{10}$. Thus, Chen's comments are based on a misinterpretation of the MCP, and various additional constraints in the future will help place the proposed MCP framework in a quantitative context. 


\section{CORRESPONDENCE}

Nianzhi Jiao and Tingwei Luo are at the State Key Laboratory of Marine Environmental Sciences at Xiamen University, 361005, China.

Gerhard J. Herndl is at the University of Vienna, Althanstrasse 14, 1090 Vienna, Austria.

Dennis A. Hansell is at the Division of Marine and Atmospheric Chemistry, Rosenstiel School of Marine and Atmospheric Science, University of Miami, 4600 Rickenbacker Causeway, Miami, Florida 33149, USA.

Ronald Benner is at the University of South Carolina, Columbia, South Carolina 29208, USA.

Gerhard Kattner is at the Alfred Wegener Institute for Polar and Marine Research, Am Handelshafen 12, D-27570 Bremerhaven, Germany.

Steven W. Wilhelm is at The University of Tennessee, 1414 West Cumberland Avenue, Knoxville, Tennessee 37996, USA.

David L. Kirchman is at the University of Delaware, School of Marine Science and Policy, 222 Cannon Lab, Lewes, Delaware 19958, USA.
Markus G. Weinbauer is at the Laboratoire d'Océanographie de Villefranche, Université Pierre et Marie Curie-Paris 6 and Centre National de la Recherche Scientifique, 06230 Villefranche-sur-Mer, France.

Feng Chen is at the University of Maryland Biotechnology Institute, 701 East Pratt Street Baltimore, Maryland 21012, USA.

Faroog Azam is at the Scripps Institution of Oceanography, University of California San Diego, 9500 Gilman Drive, La Jolla California 92093, USA. Correspondence to N.J. e-mail: Jiao@xmu.edu.cn doi: 10.1038/nrmicro2386-c5

1. Jiao, N. et al. Microbial production of recalcitrant dissolved organic matter: long-term carbon storage in the global ocean. Nature Rev. Microbiol. 8, 593-599 (2010).

2. Chen, C. T. A. Microbial carbon pump: additional considerations. Nature Rev. Microbiol. 31 May 2011 (doi: 10.1038/nrmicro2386-c4).

3. Liang, C. \& Balser, T. C. Microbial production of recalcitrant organic matter in global soils: implications for productivity and climate policy. Nature Rev. Microbiol. 9, 75 (2011)

4. Benner, R. Biosequestration of carbon by heterotrophic microorganisms. Nature Rev. Microbiol. 9, 75 (2011).

5. Carlson, C. A. et al. Interactions among dissolved organic carbon, microbial processes, and community structure in the mesopelagic zone of the northwestern Sargasso Sea. Limnol. Oceanogr. 49, 1073-1083 (2004).

6. Jiao, N. Z., Tang, K., Cai, H. Y. \& Mao, Y. J. Increasing the microbial carbon sink in the sea by reducing chemical fertilization on the land. Nature Rev. Microbiol. 9, 75 (2011)

7. McCarthy, M. D. et al. Chemosynthetic origin of ${ }^{14} \mathrm{C}$-depleted dissolved organic matter in a ridge-flank hydrothermal system. Nature Geosci. 4, 32-36 (2011)

8. Hazen, T. C. et al. Deep-sea oil plume enriches indigenous oil-degrading bacteria. Science 330 , 204-208 (2010).

9. Ogawa, H., Amagai, Y., Koike, I., Kaiser, K. \& Benner, R. Production of refractory dissolved organic matter by bacteria. Science 292, 917-920 (2001).

10. Benner, R.\& Herndl, G.J in Microbial Carbon Pump in the Ocean (eds Jiao, N. Z., Azam, F. \& Sanders, S.) (American Association for the Advancement of Science, Washington DC, 2011)

Competing interests statement

The author declares no competing financial interests. 